\title{
Multi-path Stable QoS Routing for Real-time Traffic Applications in MANET
}

\author{
Ch.Niranjan Kumar \\ Senior Assistant professor, Department of CSE \\ Sumathi Reddy Institute of technology for Women
}

\author{
N.Satyanarayana,Ph.D \\ Professor, Department of CSE \\ Nagole Institute of Technology \& Science
}

\begin{abstract}
In mobile ad hoc networks (MANET), the existing routing protocols consider the routing metrics such as bandwidth, delay and link stability in partial manner. Also the real time and nonreal time traffic flows are not distinguished based on delay and bandwidth requirements. In order to overcome these issues, in this paper multipath stable QoS routing for real time traffic in MANET is proposed. In this technique, multiple paths are discovered among source and destination. Among the discovered routes, the optimal paths are selected based on bandwidth constraints, delay constraints and path stability. When any flow request is received, it is initially categorized as real time and non-real time flows where real time flows are given higher priority. For real time flows, bandwidth and delay constrained paths are chosen. For non-real time flows, the stable paths are chosen. By simulation results, the proposed approach is validated and the approach minimizes delay and overhead.
\end{abstract}

Keywords: Mobile Ad Hoc Networks (MANET), Quality of Service (QoS), Routing.

\section{INTRODUCTION}

\subsection{Mobile AdHoc Network (MANET)}

The next generation of applications running on the wireless networks should carry diverse multimedia applications such as video, audio and data etc. Mobile Ad hoc NETworks (MANETs) are wireless mobile networks formed spontaneously. MANET is a collection of mobile nodes that can communicate with each other at multiple hops without having any central infrastructure.

Since MANET is characterized as a decentralized network; communication between any two nodes requires multi-hop relays to act as routers. Every node in MANET is permitted to move arbitrarily in the network.

In MANET, all nodes are treated equivalently and so every node has the ability to transfer data between any source and destination pairs. In this manner, every node can behave as a source or destination or router. This network is used in military actions, disaster rescues and in delay sensitive applications for the network is flexible. MANET offers Quality of Service (QoS) considering bandwidth and delay sensitive applications. The dynamic nature of MANET that connected with conditions like limited bandwidth and battery power challenges routing and facilitating QoS.

Development in multimedia technology and many civilian applications achieve QoS in MANET. Conversely, unique features of MANET such as multi-hop routing, arbitrary movement of mobile nodes, etc brings in vast number of overheads during route discovery and route maintenance phases [1] [2] [3].

\subsection{QoS in MANET}

The performance agreement between the service provider and user applications, which is described qualitatively or quantitatively considering connection requirements is defined as Quality of Service (QoS). Bandwidth, delay, jitter, loss ratio, and so on are the QoS requirements of a connection. These requirements can also be described as QoS metrics and they can concave or additive [4].

In other words, QoS can be described as the level or grade of services that the network assures. QoS and QoS parameters depend on specific requirements of applications [5] [6]

\subsection{Routing in MANET}

MANETs are made up of mobile devices that use wireless transmission for communication. A user can move anytime in an ad hoc scenario and, as a result, such a network needs to have routing protocols which can adopt dynamically changing topology. MANETs are dynamic distributed systems, in which any node can become a source or destination for a communication session and all nodes are considered as peers.

MANET offers multi-hop communication for mobile nodes are bounded with limited transmission range. Thus, to transmit data through multiple intermediate nodes, the source selects the route either proactively or reactively. Proactive routing protocol uses periodic link state updates in order to discover routes between a source and destination pair. It does not consider the requirement of network. On the other hand, reactive routing protocol discovers the route when it is needed in an on-demand manner using a broadcast query or reply cycle [7] [8].

\subsection{QoS Issues in MANET}

- Limited resource availability, dynamic topology, inadequate state information, decentralized coordination and hidden terminal problem are the important challenges in AdHoc networks.

- Routing is a key issue in providing QoS guarantee in MANET.

- Signals collide with each other in Hidden node problem.

- Network throughput is significantly reduced if the traffic load is high in hidden and exposed terminal problem.

- Dynamic environment and unreliability of the wireless medium lead to erroneous reception of data packets at the receiver.

- Owing to various hazardous conditions in the environment, nodes might fail at any time.

- Nodes may with draw from the network either voluntarily or when the battery of a node is drained out. 
- The discovered route may become stale or broken once the network topology is changed [9] [10] [11] [12].

\subsection{Real-time Multi-media Issues in MANET}

- Random mobility behavior in MANET causes poor route availability and has impact on network communication performance.

- Improving stability of routing schemes is a significant issue.

- When multimedia traffic is transmitted over mobile AdHoc network then it is difficult to guarantee QoS.

- QoS-aware route with throughput and delay constraints, none of them address the issue of reducing the path breakage during data transmission [10] [14] [16] [17].

\subsection{Problem Identification}

As for as real-time flows are concerned; route stability, delay and bandwidth are the essential parameters to be considered for routing.

In [10], a cross-layer TDMA-based routing protocol was proposed to meet the delay and bandwidth requirements for network throughput while the stability of routing protocol is not considered.

In [13], model-based QoS routing scheme was proposed to develop effective bandwidth but delay and stability are not considered.

In [15], a multi-path source routing protocol for effective bandwidth and reliability but delay is not considered.

In [16], cross-layer design increases the stability and delay but bandwidth is not considered.

In [17], with Greedy-based backup routing protocol the stability of data link and route link is maintained but delay and bandwidth are not considered.

Though stability, delay and bandwidth are discussed in [14] for the routing, it does not distinguish real time flows and non real time flows for bandwidth and delay requirements. Moreover, it applies single path routing only.

To offer an ideal solution that alleviates the above described problems, in this paper a multi-path QoS routing for real-time traffic is proposed, which considers stability, delay and bandwidth as the parameters.

\section{RELATED WORKS}

David Espes and Zoubir Mammeri [10] have presented a cross-layer TDMA-based routing protocol. Their main objective is to endure delay and bandwidth requirements while optimizing network throughput. They have proposed a QoS routing protocol to be utilized in TDMA-based MANETs. Their protocol selects paths considering impact factor of a node. Priority will be given to a route with a low impact on the network. The impact factor is the amount of bandwidth consumed by admitted flows. When the network load increases, their protocol allows more flows. The improvement of network throughput comes with a cost. This protocol has a higher overhead than QoS-AODV. Finally, it should be noticed that this protocol is more scalable than QoS-AODV and AODV. It is particularly efficient in dense environments where MANET may be deployed.

Atef Abdrabou and Weihua Zhuang [13] have put forwarded a model-based quality-of-service $(\mathrm{QoS})$ routing scheme for
IEEE 802.11 ad hoc networks. Their scheme offers stochastic end-to-end delay guarantees, instead of average delay guarantees, to delay-sensitive bursty traffic sources. Using a cross-layer design approach, their scheme selects the routes based on a geographical on-demand ad hoc routing protocol and verifies the availability of network resources by using traffic source and link-layer channel modeling. It has considered the IEEE 802.11 characteristics and node interactions. Their scheme enhances the effective bandwidth theory and its dual effective capacity concept to multi-hop IEEE 802.11 ad hoc networks

A Route Stability based QoS Routing (RSQR) protocol for Mobile Ad Hoc Networks (MANETs) is proposed by Nityananda Sarma and Sukumar Nandi [14]. Their work is an enhancement of QoS routing with throughput and delay conditions. Guaranteeing valid data path for sufficiently longer period of time is a very daunting problem in MANET owing to its highly dynamic nature. They have proposed a simple model for computing link stability and route stability based on received signal strengths. By adding some additional fields in route request/reply packets, the route stability information can be used to choose a route with higher stability among all the feasible routes between a source destination pair.

Fujian Qin and Youyuan Liu [15] have proposed a multi-path source routing protocol. They have considered bandwidth and reliability as QoS metrics. During routing discovery phase, the protocol chooses several multiple alternate paths that meet the QoS requirements and the ideal number of multi-path routing. The paths are selected to satisfy the tradeoff between load balancing and network overhead. During route maintenance phase, their scheme effectively handles with route failures as like DSR. Furthermore, the per-packet granularity is adopted in traffic allocation phase.

T. Quazi et al. [16] have proposed a Cross-layer design. Their system offers a dynamic resource allocation where more than one or all three traffic classes can be communicated at the same time in one frame. They have introduced their design with not only the intention of providing $\mathrm{QoS}$ in a multimedia wireless system, but also makes the resource allocation process more efficient. Their technique will increase data link layer throughput and lower average delays. However, their crosslayer design increases the stability and delay but bandwidth is not considered.

Wenjing Yang et al [17] have proposed a Greedy-based backup Routing (GBR) protocol. Their protocol has considered both route length and link lifetime in order to achieve high route stability. In their GBR, the primary path is constructed based on a greedy forwarding mechanism, whereas the local-backup path for each link is determined according to the link life-time. Nevertheless, with Greedy-based backup routing protocol the stability of data link and route link is maintained but delay and bandwidth are not considered.

\section{PROPOSED SOLUTION}

\subsection{Overview}

In this paper, multipath stable QoS routing for real time traffic in MANET is proposed. In this technique, multiple paths are discovered among source and destination nodes. Among the discovered routes, the optimal paths are selected based on bandwidth constraints, delay constraints and path stability. Here bandwidth constraints refer to the path with maximum bandwidth and delay and delay constraints refers to the path with minimum delay and bandwidth. When any flow request is received, it is initially categorized as real time and non-real time 
flows. Real-time flow is given more priority to non-real time flow. For real time flows, bandwidth and delay constrained paths are chosen based on requirement. For non-real time flows, the stable paths are chosen.

\subsection{Estimation of Bandwidth, Delay and Stability}

Let $\mathrm{z}$ represent the link which has its relative bandwidth, delay and stability.

$\mathrm{z}=(\mathrm{x}, \mathrm{y})$ be in $\mathrm{Z}$

where $\mathrm{Z}=$ set of links connecting the nodes $\mathrm{x}$ and $\mathrm{y}$ are randomly considered nodes

Let $\mathrm{V}_{\mathrm{i}}$ be the path among $\mathrm{x}$ and $\mathrm{y}$

i.e. $\mathrm{V}(\mathrm{x}, \mathrm{y})=\left\{\mathrm{V}_{0}, \mathrm{~V}_{1}, \mathrm{~V}_{2}, \ldots, \mathrm{V}_{\mathrm{n}}\right\}$

Let $A B$ be the available bandwidth for path $V_{i}$

Let $\mathrm{d}$ be the delay for $\mathrm{V}_{\mathrm{i}}$

Let $\mathrm{Q}$ be the link stability for $\mathrm{V}_{\mathrm{i}}$

The available bandwidth at time interval $t$ is defined as the minimum of the available bandwidth of all links $\mathrm{Z}$ for the path $\mathrm{V}_{\mathrm{i}}$. Each node determines the available bandwidth by subtracting the consumed cumulative bandwidth in the interference range of the node from the raw bandwidth.

$$
\mathrm{AB}\left(\mathrm{V}_{\mathrm{i}}\right)=\min \left\{\mathrm{AB}\left(\mathrm{z}_{0}\right), \mathrm{AB}\left(\mathrm{z}_{1}\right), \ldots \ldots, \mathrm{AB}\left(\mathrm{z}_{\mathrm{n}}\right)\right\}
$$

where $\mathrm{z}_{0}, \mathrm{z}_{1}, \ldots, \mathrm{z}_{\mathrm{n}}$ are the edges creating the path.

The delay is estimated based on the round trip time and queuing delay. For path $\mathrm{V}_{\mathrm{i}}$, the delay is defined as the sum of delay experienced at each link $\mathrm{z}$.

$$
\mathrm{d}\left(\mathrm{V}_{\mathrm{i}}\right)=\sum_{z \in V_{i}} d(z)
$$

The link stability for the path $\mathrm{V}_{\mathrm{i}}$ depends on the received signal strength which is mathematically defined using following equation.

$$
\mathrm{Q}\left(\mathrm{V}_{\mathrm{i}}\right)=\prod_{z \in V_{i}} Q(z)
$$

\subsection{Differentiating the Traffic Class}

Practically, it is difficult to separate the different traffic classes in MANET. However it is possible to offer relative priorities. It involves the provision of higher priority or lower priority for different traffic classes. In this technique, a token bucket model is considered for prioritizing two traffic classes such as real time and non-real traffic classes. Real time traffic belongs of delay sensitive category and non-real time traffic is insensitive. Hence real time traffic is given high priority than non-real time traffic. The token bucket model is explained below. [18]
In this model, the service profile maintains the strategies to distinguish long and short flows and core network helps in scheduling the packet removal from the queue.

The token bucket service profile takes the following three parameters into consideration.

i) Average rate: It denotes the average rate at which a packet is forwarded into the network.

ii) Peak rate: It denotes the maximum rate at which the packets are transmitted within minimum duration.

iii) Burst size: It denotes the maximum number of packets that are transmitted in minimum duration.

If the incoming packet matches the service profile

Then

Incoming packet is marked with high priority and allowed through queue-in.

Else

Incoming packet is marked with lower priority and allowed though queue-out.

End if

\subsection{Multi-path Stable QoS Routing}

The proposed scheme involves establishing multiple stable QoS routes for real time traffic. This involves the following phases

1) Multiple Route Discovery

2) Optimal path selection based on the incoming traffic

\section{Phase 1 Multipath Route Discovery}

Let $\mathrm{S}$ and $\mathrm{D}$ be the source and destination node respectively Let RREQ and RREP be the route request and route reply message respectively.

Let DP be the data packet

Let $A_{\text {th }}, d_{t h}$ and $Q_{t h}$ be the pre-defined threshold of $A B, d$, and Q.

The steps involved in the multiple path route discovery are as follows

1) When the nodes are deployed in the network, they periodically broadcast the HELLO message to its neighbor nodes. The HELLO message includes the node ID and its location information.

2) Based on the HELLO messages, each node identifies itself and also maintains the neighbors list which contains the nodes ID and location of each neighbor node.

3) When $S$ wants to transmit DP to D, a RREQ message is transmitted to $\mathrm{D}$. This is performed using modified MAC scheme. This reveals that though RREQ is transmitted to the destination at the routing layer in unicast manner, it acts as a broadcast frame at the MAC layer.

i.e. When RREQ is unicasted towards $\mathrm{D}$, it is received by every intermediate node $\mathrm{N}_{\mathrm{i}}$.

The format of RREQ message is as follows 


\begin{tabular}{|c|c|c|c|c|c|c|}
\hline \multicolumn{7}{|c|}{ Table - 1 Format of RREQ message } \\
\hline $\begin{array}{c}\text { Source } \\
\text { Node } \\
\text { ID }\end{array}$ & $\begin{array}{c}\text { Destinati } \\
\text { on ID }\end{array}$ & $\begin{array}{c}\text { Previous } \\
\text { Hop } \\
\text { node ID }\end{array}$ & $\begin{array}{c}\text { Location } \\
\text { informatio } \\
\mathrm{n}\end{array}$ & Bandwidth & $\begin{array}{c}\text { Dela } \\
\mathrm{y}\end{array}$ & Stability \\
\hline
\end{tabular}

1) $N_{i}$ upon receiving RREQ packet, updates its routing table with the information that includes source ID, destination ID, Previous hop node ID, location, bandwidth, delay and link stability (Estimated in section 3.2.1). It appends its state in the node state field of RREQ message and analyzes the destination ID. (Note-2 describes the routing table contents of the node)

$$
\text { 2) If } \mathrm{N}_{\mathrm{i}} \neq \mathrm{N}_{\mathrm{i}+1} \| \mathrm{N}_{\mathrm{i}} \neq \mathrm{N}_{\mathrm{i}-1}
$$

Then

$$
\text { 3) If } \mathrm{N}_{\mathrm{i}}=\mathrm{N}_{\mathrm{i}+1} \text { End if } \mathrm{N}_{\mathrm{i}} \text { discards RREQ }
$$

Then

$\mathrm{N}_{\mathrm{i}}$ computes $\mathrm{AB}, \mathrm{d}, \mathrm{Q}$ and adds the reverse path to the primary path table.

$$
\text { 4) If } \mathrm{N}_{\mathrm{i}+1} \neq \mathrm{D}
$$

Then

$\mathrm{N}_{\mathrm{i}+1}$ adds $\mathrm{AB}, \mathrm{d}$, Q to RREQ packet and transmits it to MAC layer

End if

5) When D receives RREQ packet, RREP message is sent back to $S$ in the path taken by RREQ in reverse direction.

6) $\quad \mathrm{S}$ and $\mathrm{N}_{\mathrm{i}}$ upon receiving RREP sets the path to $\mathrm{D}$ based on the following condition.

$$
\text { 1) If }\left(\mathrm{Q}_{\mathrm{i}}<\mathrm{Q}_{\mathrm{th}}\right)
$$

Then

End if

$$
\text { Corresponding path is rejected. }
$$

2) If $\left[\left(\mathrm{AB}_{\mathrm{i}}>\mathrm{AB}_{\mathrm{th}}\right) \&(\mathrm{di}>\mathrm{dth}) \&\left(\mathrm{Q}_{\mathrm{i}}>\mathrm{Q}_{\mathrm{th}}\right)\right]$

Then

The corresponding paths chosen as primary paths End if

3) If any primary path fails to satisfy routing constraints or encounters failure

Then

$S$ uses the next level of the $A B_{i}, d_{i}$ and $Q_{i}$, from the collected information of subsequent RREP in deciding the next local backup paths.

End if

Note -2: Each node maintains a data structure which includes sub-tables.

i. Multiple routing table: It contains the multiple routes information for $\mathrm{D}$

ii. RREQ table: This table is illustrated in table $-1$

iii. Data cache: It stores the latest DPs which are forwarded recently.

Figure 1 demonstrates the route request and reply process. $\mathrm{N}_{1}$ and $\mathrm{N}_{9}$ act as the source and destination respectively. Four paths $\mathrm{V}_{1}\left[\mathrm{~S}-\mathrm{N}_{5}, \mathrm{~N}_{4}, \mathrm{~N}_{15}, \mathrm{~N}_{8}-\mathrm{D}\right], \mathrm{V}_{2}\left[\mathrm{~S}, \mathrm{~N}_{3}, \mathrm{~N}_{6}, \mathrm{~N}_{7}, \mathrm{~N}_{12}, \mathrm{D}\right], \mathrm{V}_{3}\left[\mathrm{~S}, \mathrm{~N}_{2}, \mathrm{~N}_{10}\right.$, $\left.\mathrm{N}_{12}-\mathrm{D}\right]$ and $\mathrm{V}_{4}\left[\mathrm{~S}, \mathrm{~N}_{13}, \mathrm{~N}_{14}, \mathrm{~N}_{11}, \mathrm{D}\right]$ are established.

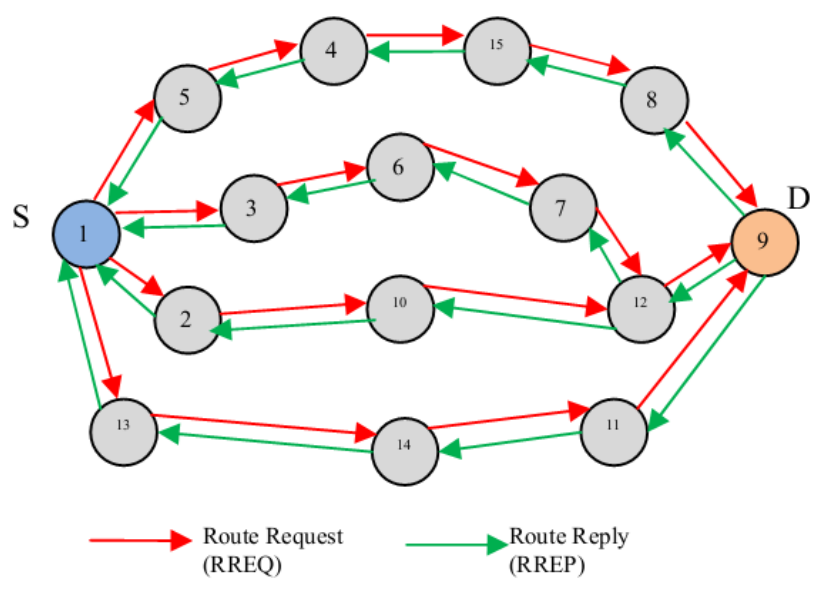

Figure 1: Route Discovery process

Phase 2: Optimal Route selection based on incoming traffic. When any flow request is received, it is categorized as real-time and non-real time flow. Real-time flow is given more priority to non-real time flow.

For real time flows, two parameters are taken into consideration.

\section{1) Bandwidth constrained parameter:}

This parameter requires maximum bandwidth with maximum delay.

\section{2) Delay constrained parameter:}

This parameter requires minimum delay with minimum required bandwidth

The multiple paths which are selected in phase-1 are categorized as follows

(i) Stable paths

(ii) Paths with required bandwidth and stability

(iii) Paths with required delay and stability.

\section{Real time traffic}

When flow request corresponds to bandwidth constrained real time traffic, then paths in category (ii) are selected.

When the flow request corresponds to delay constrained real time traffic, then the paths in category (iii) are selected.

\section{Non-real time traffic}

For non-real time flows, the paths in category (i) are selected.

Figure 2 demonstrates the optimal route selection for real time and non-real time traffic application. Since, $V_{1}$ and $V_{2}$ contains maximum bandwidth and delay and maximum stability, it is suitable for bandwidth constrained real time traffic. As, $\mathrm{V}_{3}$ contains minimum delay, required minimum bandwidth and maximum stability, it is suitable for delay constrained real time traffic. Since the link stability of the $\mathrm{V}_{4}$ contains maximum received signal strength, it is said to have maximum stability. Hence it is suitable for non-real time traffic flow. 


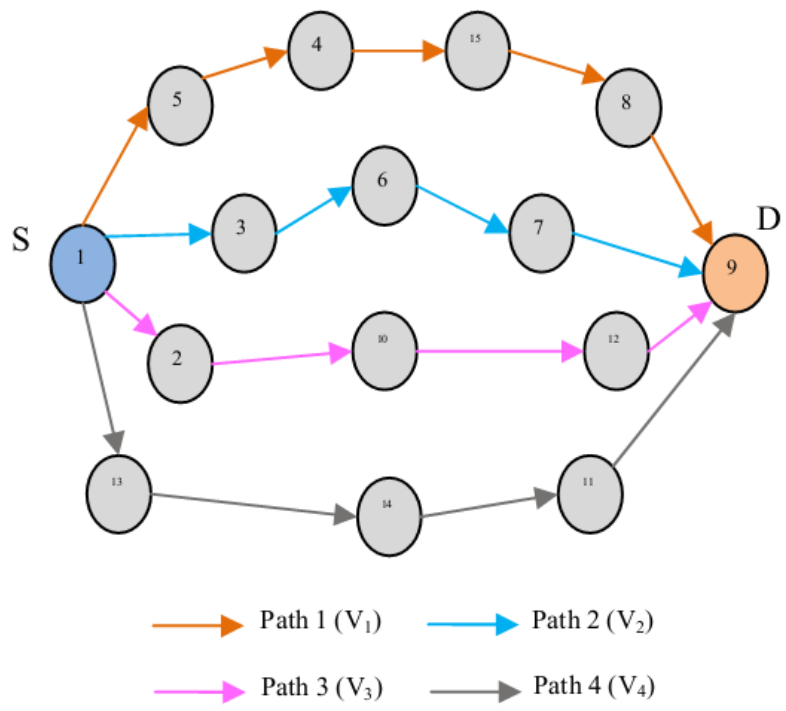

Figure 2: Optimal Route selection based on incoming traffic

\section{SIMULATION RESULTS}

\subsection{Simulation Parameters}

The Multi-path Stable QoS Routing (MSQR) scheme is evaluated through NS-2 [19]. A bounded region of $1000 \times 1000$ sqm is considered, in which the nodes are distributed uniformly. The number of nodes is varied as 50,100,150 and 200.In simulation, the channel capacity of mobile hosts is set to the same value: $2 \mathrm{Mbps}$. The distributed coordination function (DCF) of IEEE 802.11 for wireless LANs is used as the MAC layer protocol. The simulated traffic is Constant Bit Rate (CBR).

The following table summarizes the simulation parameters

Table 2: Simulation parameters

\begin{tabular}{|l|l|}
\hline No. of Nodes & $50,100,150$ and 200. \\
\hline Area Size & $1000 \times 1000$ \\
\hline Mac & 802.11 \\
\hline Simulation Time & $50 \mathrm{sec}$ \\
\hline Traffic Source & CBR \\
\hline Packet Size & 512 \\
\hline Transmission Rate & $250 \mathrm{~m}$ \\
\hline Routing Protocol & MSQR \\
\hline Rate & $250 \mathrm{~Kb}$ \\
\hline Speed & $5,10,15,20$ and $25 \mathrm{~m} / \mathrm{s}$ \\
\hline
\end{tabular}

\subsection{Performance Metrics}

The performance of our proposed MSQR approach is compared with Greedy based backup Routing (GBR) [17] technique. The evaluation is performed mainly based on the following metrics:

Received Bandwidth: It is the number of bits transmitted to the receiver.

Throughput: It is the number of packets received by the receiver during the transmission.

End-to-End-Delay: It is the amount of time taken by the packet to reach the destination.
The simulation results are presented in the next section.

\subsection{Results \& Analysis}

Case 1: (CBR)

\section{A. Based on Nodes}

In the first experiment the metrics are analyzed by varying the number of nodes as 50,100,150 and 200 .

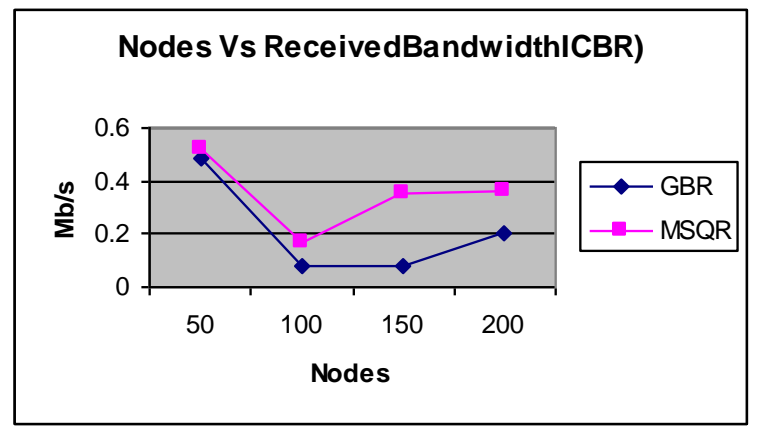

Figure 3: Nodes Vs Received bandwidth

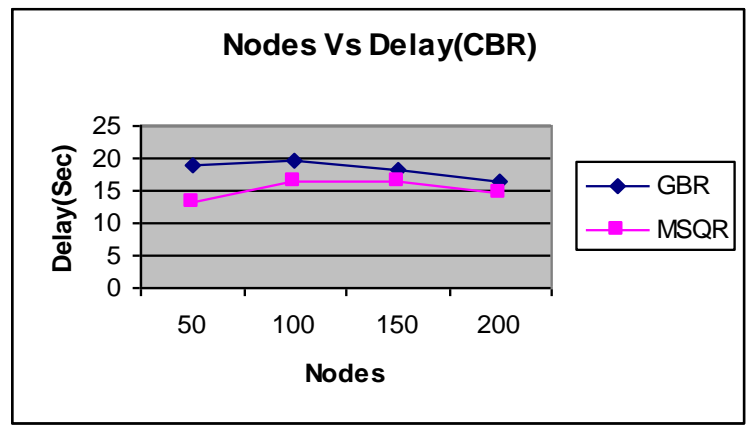

Figure 4: Nodes Vs Delay

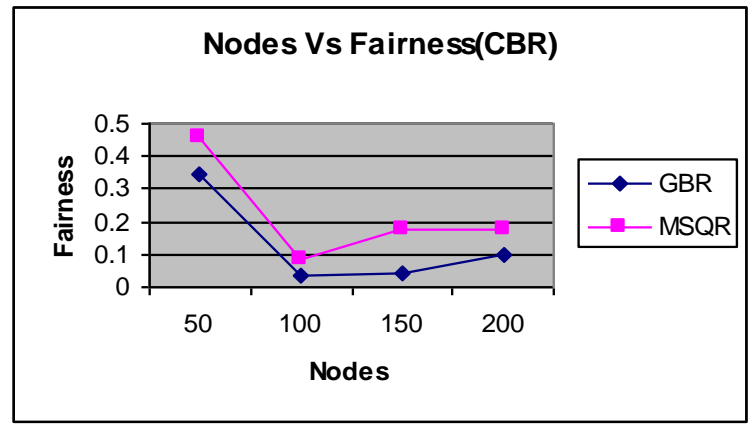

Figure 5: Nodes Vs Fairness

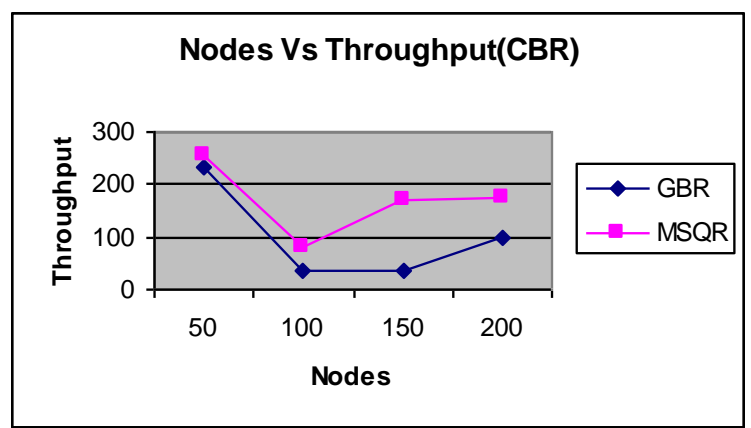


Figure 6: Nodes Vs Throughput

In figure 3 , it is observable that the received bandwidth of our proposed MSQR is better than the existing GBR technique.

In figure 4 , it is obvious that the delay of our proposed MSQR is less than the existing GBR technique.

In figure 5, it is visible that the fairness of our proposed MSQR is higher than the existing GBR technique.

In figure 6, it is noticeable that the throughput of our proposed MSQR is higher than the existing GBR technique.

\section{B. Based on Speed}

In second experiment, the mobile speed is varied as $5 \mathrm{~m} / \mathrm{s}$ to $25 \mathrm{~m} / \mathrm{s}$.

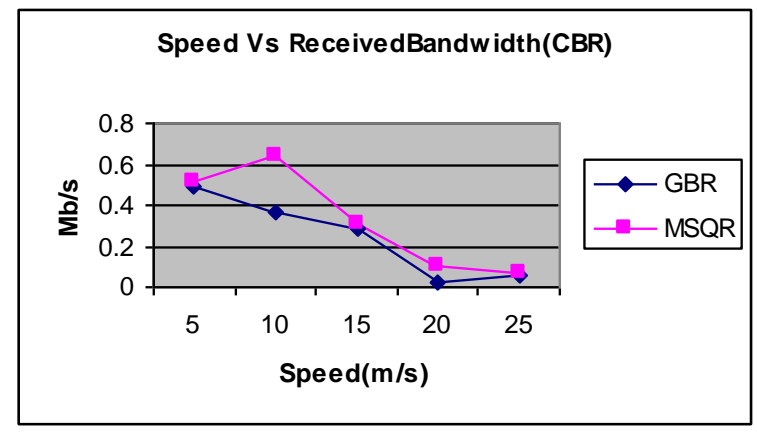

Figure 7: Speed Vs Received Bandwidth

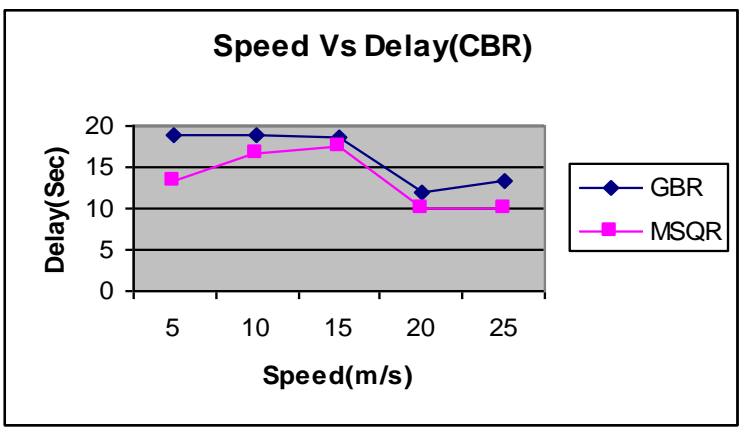

Figure 8: Speed Vs Delay

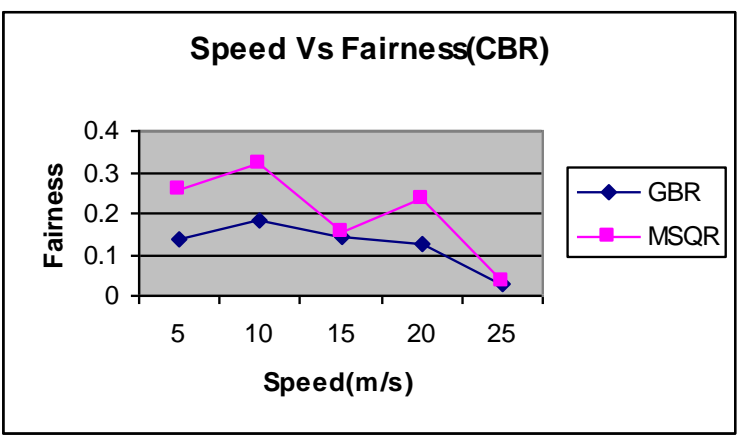

Figure 9: Speed Vs Fairness

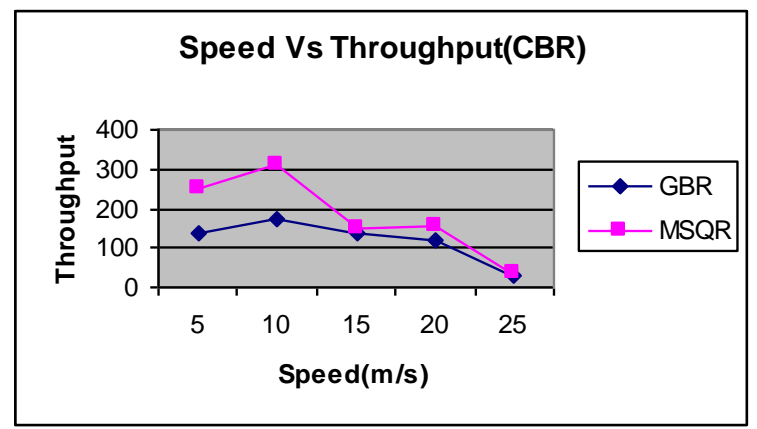

Figure 10: Speed Vs Throughput

In figure 7 , it is obvious that the received bandwidth of our proposed MSQR is better than the existing GBR technique.

In figure 8 , it is observable that the delay of our proposed MSQR is less than the existing GBR technique.

In figure 9, it is noticeable that the fairness of our proposed MSQR is higher than the existing GBR technique.

In figure 10 , it is visible that the throughput of our proposed MSQR is higher than the existing GBR technique.

\section{Case -2 (Video)}

A. Based on Nodes

In third experiment, the number of nodes is varied as $50,100,150$ and 200 .

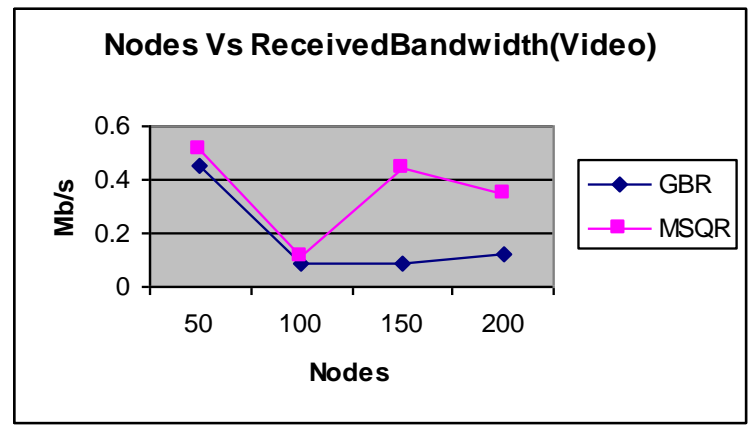

Figure 11: Nodes Vs Received Bandwidth

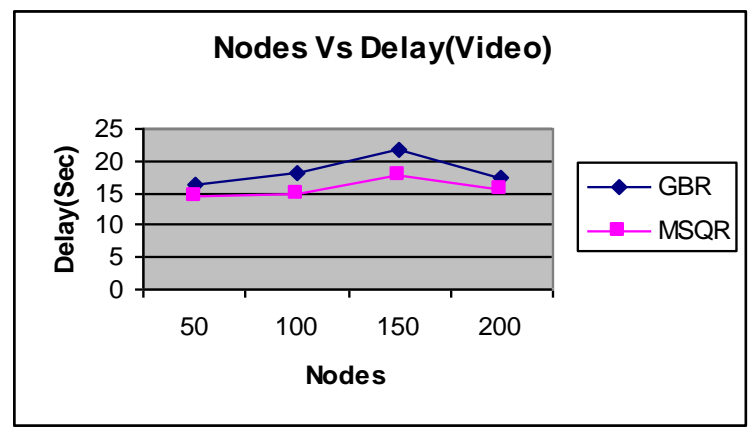

Figure 12: Nodes Vs Delay 


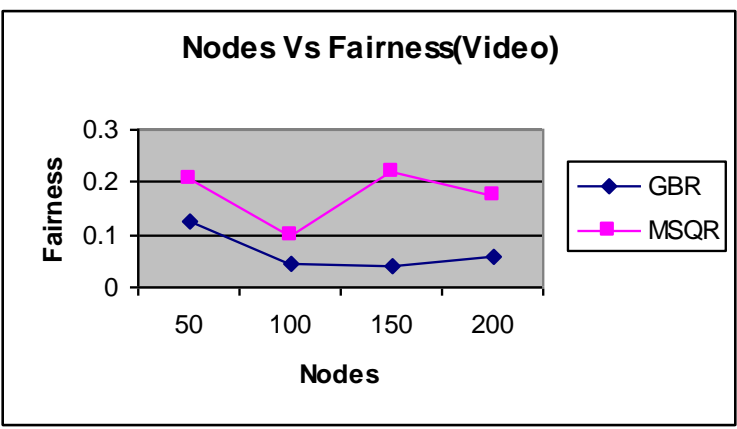

Figure 13: Nodes Vs Fairness

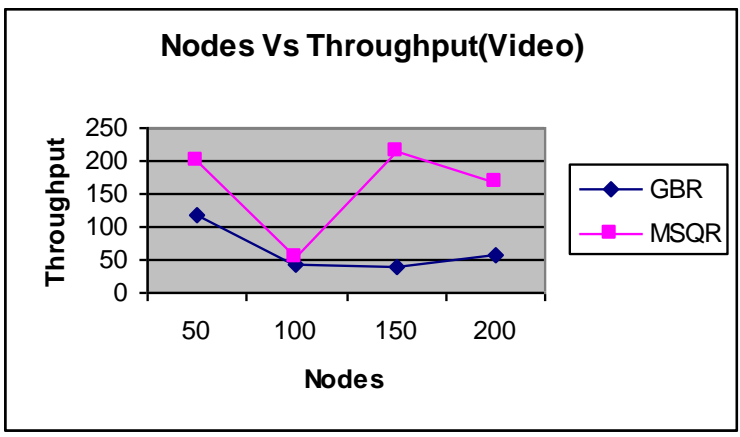

Figure 14: Nodes Vs Throughput

In figure 11, it is noticeable that the received bandwidth of our proposed MSQR is better than the existing GBR technique.

In figure 12 , it is obvious that the delay of our proposed MSQR is less than the existing GBR technique.

In figure 13, it is visible that the fairness of our proposed MSQR is higher than the existing GBR technique.

In figure 14, it is observable that the throughput of our proposed MSQR is higher than the existing GBR technique.

\section{B. Based on Speed}

In fourth experiment, the node's speed is varied as $5 \mathrm{~m} / \mathrm{s}$ to $25 \mathrm{~m} / \mathrm{s}$.

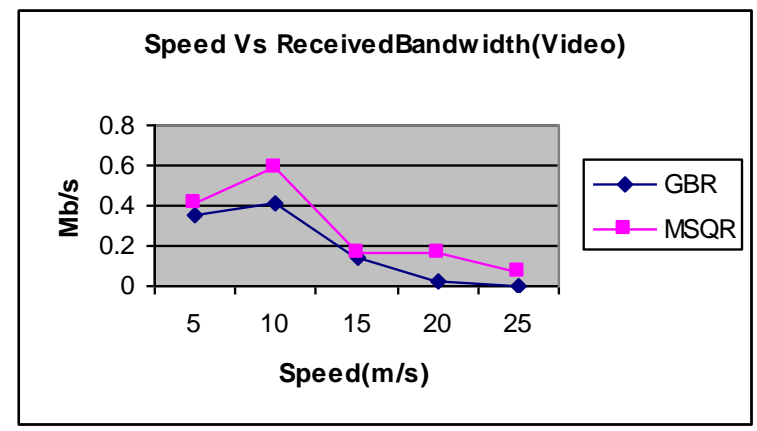

Figure 15: Speed Vs Received Bandwidth

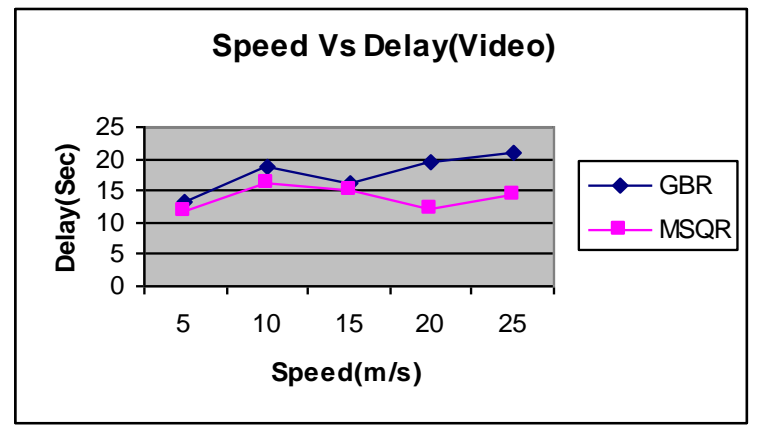

Figure 16: Speed Vs Delay

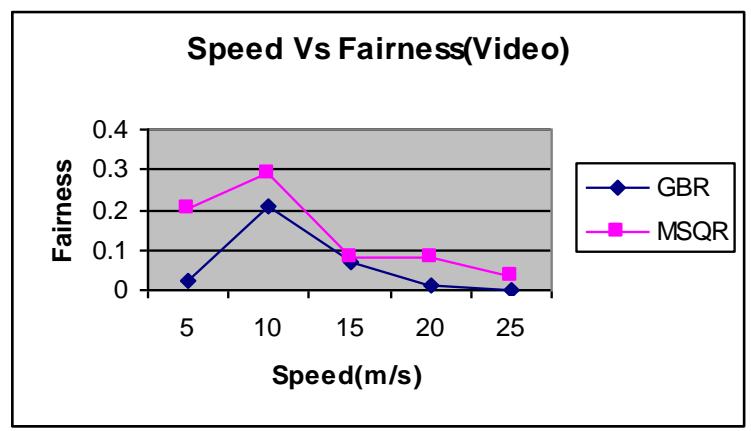

Figure 17: Speed Vs Fairness

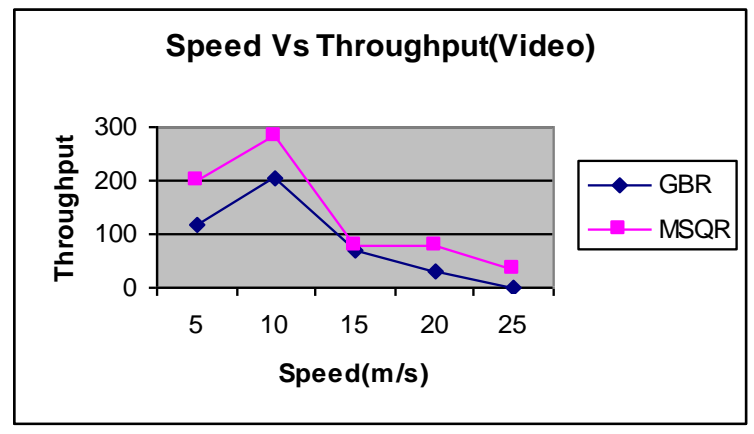

Figure18: Speed Vs Throughput

In figure 15 , it is noticeable that the received bandwidth of our proposed MSQR is better than the existing GBR technique.

In figure 16, it is visible that the delay of our proposed MSQR is less than the existing GBR technique.

In figure 17 , it is obvious that the fairness of our proposed MSQR is higher than the existing GBR technique.

In figure 18 , it is observable that the throughput of our proposed MSQR is higher than the existing GBR technique.

\section{CONCLUSION}

In this paper, multipath stable QoS routing for real time traffic in MANET is proposed. In this technique, multiple paths are discovered among source and destination. Among the discovered routes, the optimal paths are selected based on bandwidth constraints, delay constraints and path stability. Here bandwidth constraints refer to the path with maximum bandwidth and delay and delay constraints refers to the path with minimum delay and bandwidth. When any flow request is received, it is initially categorized as real time and non-real time flows. Real-time flow is given more priority to non-real time 
flow. For real time flows, bandwidth and delay constrained paths are chosen based on requirement. For non-real time flows, the stable paths are chosen. By simulation results, the proposed approach is simulated and it minimizes delay and overhead.

\section{REFERENCES}

[1] Prof. P.K. Suri, Dr. M. K.Soni and Parul Tomar, "Cluster Based QoS Routing Protocol for MANET", International Journal of Computer Theory and Engineering, Vol. 2, No. 5, October, 2010.

[2] Zhenyu Liu, Marta Z. Kwiatkowska, and Costas Constantinou, "A Biologically Inspired QoS Routing Algorithm for Mobile Ad Hoc Networks", International Journal Of Wireless And Mobile Computing (IJWMC), 2006.

[3]Muhammad O. Farooq and Ghalib A. Shah, "A Reactive QoS Routing Protocol For MANET", Ad Hoc \& Sensor Wireless Networks, Vol. 13, pp. 13-38 2011

[4]Ying Ge, "Quality-of-Service Routing in Ad-Hoc Networks Using OLSR", technical paper, December 2002.

[5]Ash Mohammad Abbas and Oivind Kure, "Quality of Service in mobile ad hoc networks: a survey", Int. J. Ad Hoc and Ubiquitous Computing, Volume 6 Issue 2, July 2010.

[6]Navid Nikaein and Christian Bonnet, "A Glance at Quality of Service Models for Mobile Ad Hoc Networks", In Proc. of DNAC, December 2002

[7]Vahid Ayatollahi Tafti, Abolfazl Gandomi, "Performance of QoS Parameters in MANET Application Traffics in Large Scale Scenario", World Academy of Science, Engineering and Technology 722010

[8]Natarajan Meghanathan, "Exploring the Performance Tradeoffs among Stability-Oriented Routing Protocols for Mobile Ad hoc Networks", Network Protocols and Algorithms, ISSN 1943-3581, 2010, Vol. 2, No. 3.

[9] G. Usha Devi and G. Kavitha, "Resource Reservation using PRTMAC Protocol in MANET", IRACST - International Journal of Computer Networks and Wireless Communications (IJCNWC), ISSN: 2250-3501 Vol.2, No.3, June 2012.

[10] David Espes, Zoubir Mammeri, "Delay and Bandwidth constrained Routing with Throughput optimization in TDMA-based MANETs", 2009 IEEE

[11] Gurpinder Singh, Asst. Prof. Jaswinder Singh, "MANET: Issues and Behavior Analysis of Routing Protocols", International Journal of Advanced Research in Computer
Science and Software Engineering, Volume 2, Issue 4, April 2012.

[12]Stephen Mueller, Rose P. Tsang, and Dipak Ghosal, "Multipath Routing in Mobile Ad Hoc Networks: Issues and Challenges", Performance Tools and Applications to Networked Systems, pp. 209-234, Springer, 2004.

[13]Atef Abdrabou and Weihua Zhuang, "Statistical QoS Routing for IEEE 802.11 Multihop Ad Hoc Networks", IEEE Transactions On Wireless Communications, Vol. 8, No. 3, March 2009.

[14]Nityananda Sarma and Sukumar Nandi, "Route Stability Based QoS Routing in Mobile Ad Hoc Networks", Wireless Personal Communications Volume 54, Issue 1, pp 203-224, 2010

[15]Fujian Qin and Fujian Qin, "Multipath Based QoS Routing in MANET", Journal of Networks, Vol. 4, No. 8, October 2009.

[16]T. Quazi H.J. Xu and F. Takawira, "Quality of service for multimedia traffic using cross-layer design", IET Communication., 2009, Vol. 3, Iss. 1, pp. 83-90.

[17]Wenjing Yang, Xinyu Yang, Shusen Yang and Dongxu Yang, "A Greedy-based Stable Multi-path Routing Protocol in Mobile Ad hoc Networks", Journal Ad Hoc Networks archive, Volume 9 Issue 4, June, 2011

[18]Nandhini Sivasubramaniam and Palaniammal Senniappan, "Stateless Aggregate Fair Marking Scheduler For Differentiated Service Networks", Journal of Computer Science, pp 63-73, 2013.

[19] Network Simulator: http://www.isi.edu/nsnam/ns/

Chukka Niranjan Kumar, B.E., M.Tech, (Ph.D.), MIEEE, MISTE, MIETE. Currently, he is a Senior Assistant professor in CSE department in Sumathi Reddy Institute of technology for Women. His specializations include networking, MANET, Network Security. His current research interests are wireless communications and networking, MANET, Network Security,

Dr.N.Satyanarayana, M.Sc, M.Phil, AMIE (ET), M.Tech (CS), Ph.D (CSE), MISTE, MCSI, received his Ph.D degree in Computer Science \& Engineering from Acharya Nagarjuna University, currently working as a Professor in department of CSE at Nagole Institute of Technology \& Science. His research interests include Advanced Computer Architecture, Networking, and Wreless Communications 